\title{
One-pot synthesis of stable phosphorus ylides using aldehyde phenylhydrazone derivatives
}

\author{
Reza Heydari, Nourallah Hazeri, Malek Taher Maghsoodlou, Sayyed Mostafa Habibi \\ Khorassani, Farahnaz Barahuie, and Ghasem Marandi
}

Department of Chemistry, The University of Sistan and Baluchestan

P. O. Box 98135-674, Zahedan, Iran

E-mail:

\begin{abstract}
Stable crystalline phosphorus ylides were obtained in excellent yields from the 1:1:1 addition reaction between triphenylphosphine and dialkyl acetylenedicarboxylates in the presence of phenylhydrazone derivatives, such as 1-benzylidene-2-phenylhydrazine, 1-(3chlorobenzylidene)-2-phenylhydrazine, 1-(2,4-dinitrobenzylidene)-2-phenylhydrazine, 1-(4nitrobenzylidene)-2-phenylhydrazine, 1-(2-fluorobenzylidene)-2-phenylhydrazine, 1-(4fluorobenzylidene)-2-phenylhydrazine and 1-(2,4-dichlorobenzylidene)-2-phenylhydrazine. These stable ylides exist in solution as a mixture of two geometrical isomers as a result of the restricted rotation around the carbon-carbon partial double bond resulting from the conjugation of the ylide moiety with the adjacent carbonyl group.
\end{abstract}

Keywords: Acetylenic ester, phenylhydrazone derivatives, geometrical isomers, stable phosphorus ylides, triphenylphosphine

\section{Introduction}

The impact of organophosphorus compounds chemistry on modern synthetic chemistry is difficult to quantify, but one can safely assume that the study of this element has influenced many areas of chemical endeavour. ${ }^{1-4}$ The chemistry of phosphorus ylides is very significant in the chemistry of the twentieth century. ${ }^{5}$ Phosphorus ylides have found use in a wide variety of reactions, especially in the synthesis of naturally occurring products, and compounds with biological and pharmacological activity. ${ }^{6}$ Many methods have been described involving novel synthesis of organophosphorus compounds. ${ }^{1-11}$ Phosphorous ylides are most often prepared by treatment of a phosphonium salt with a base. Phosphonium salts are usually made from a phosphine and an alkyl halide. ${ }^{1-8}$ There are many studies on the reaction between trivalent phosphorus nucleophiles and $\alpha, \beta$-unsaturated carbonyl compounds in the presence of a proton 
source such as $\mathrm{OH}, \mathrm{CH}, \mathrm{NH}$ or $\mathrm{SH}^{8-27}$ Here we describe an efficient synthetic route for the preparation of stable phosphorus ylides 3 using triphenylphosphine, dialkyl acetylenedicarboxylates $\mathbf{1}$ and phenylhydrazone derivatives such as 1-benzylidene-2phenylhydrazine, 1-(3-chlorobenzylidene)-2-phenylhydrazine, 1-(2,4-dinitrobenzylidene)-2phenylhydrazine, 1-(4-nitrobenzylidene)-2-phenylhydrazine, 1-(2-fluorobenzylidene)-2phenylhydrazine, 1-(4-fluorobenzylidene)-2-phenylhydrazine and 1-(2,4-dichlorobenzylidene)-2phenylhydrazine 2 (Scheme1).

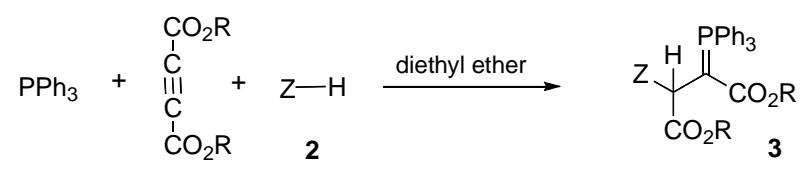

1

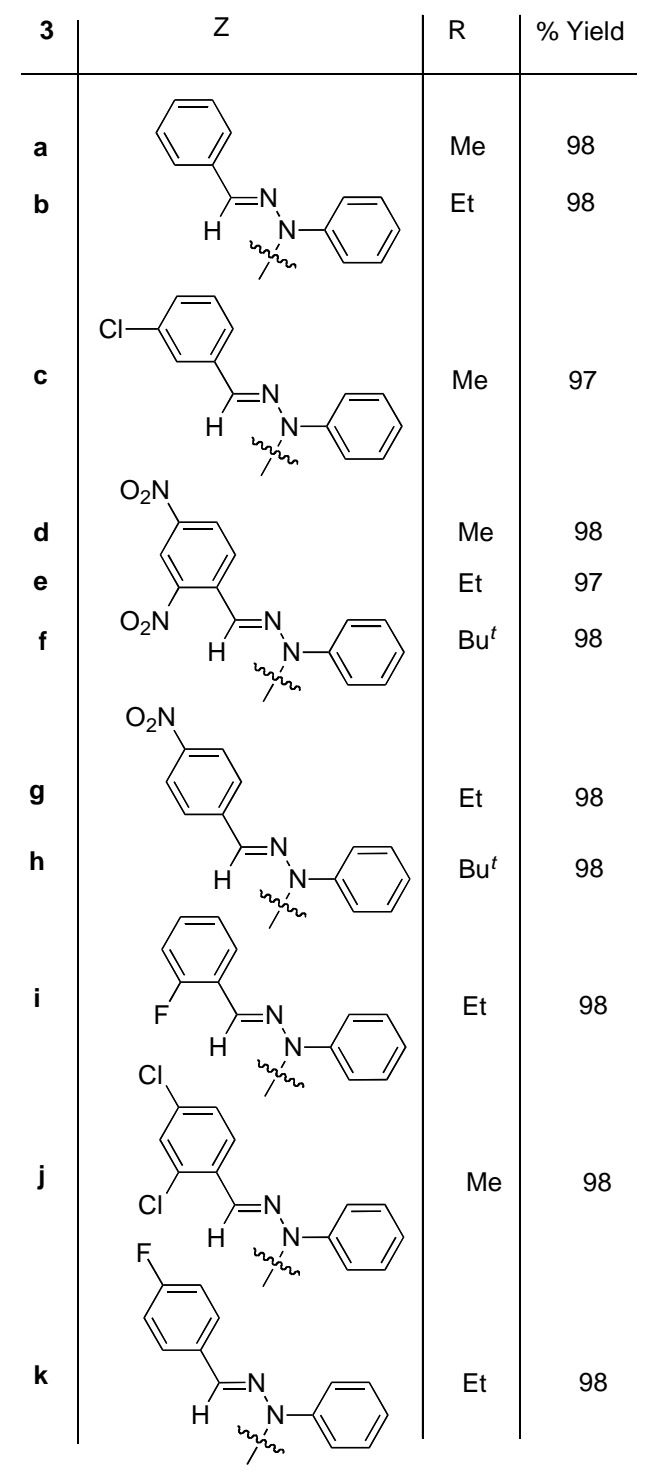

\section{Scheme 1}




\section{Results and Discussion}

The reaction between aldehyde phenylhydrazone derivatives and dialkyl acetylenedicarboxylates $\mathbf{1}$ in the presence of triphenylphosphine proceeded at ambient temperature in diethyl ether and was complete after a few hours. The ${ }^{1} \mathrm{H},{ }^{13} \mathrm{C}$ and ${ }^{31} \mathrm{P}$ NMR spectra of the crude product clearly indicated the formation of stable phosphorus ylides 3 (see Scheme 2). No product other than 3 could be detected by NMR spectroscopy. The structures of compounds 3a-k were deduced from their elemental analyses, mass, IR, ${ }^{1} \mathrm{H},{ }^{13} \mathrm{C}$, and ${ }^{31} \mathrm{P}$ NMR spectra. The mass spectra of these stable ylides displayed molecular ion peaks at appropriate $\mathrm{m} / \mathrm{z}$ values. Fragmentation involved the loss of the side-chains and a benzylidene phenylhydrazine.

The ${ }^{1} \mathrm{H},{ }^{13} \mathrm{C}$, and ${ }^{31} \mathrm{P}$ NMR spectra of ylides 3a-e, $\mathbf{3 g}$ and 3i-k showed them to be a mixture of two isomers. The ylide moiety of these compounds is strongly conjugated with the adjacent carbonyl group, and rotation about the partial double bond in (E)-3 and (Z)-3 geometrical isomers (see Scheme 2) is slow on the NMR timescale at room temperature.

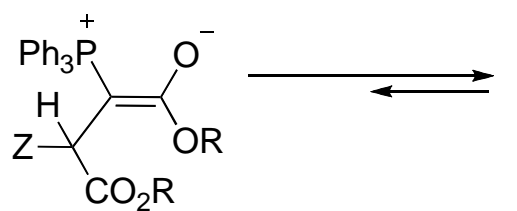

(E)-3; Minor

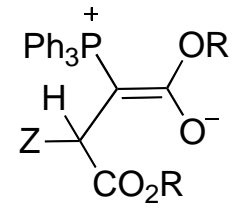

(Z)-3; Major

\section{Scheme 2}

The assignments of 3-(Z)- and the 3-(E)-isomers as the major and minor forms in phosphorus ylides have been reported previously. ${ }^{28-31}$ The ${ }^{1} \mathrm{H}$ NMR spectrum of compound 3a exhibited two singlets ( $\delta 3.06$ and 3.87) arising from the methoxy group in the Z-isomer, and two singlets at 3.42 and $3.78 \mathrm{ppm}$ for that in the E-isomer. The methyl group at 3.06 in the Z-isomer is shielded due to the anisotropic effect of a phenyl group of the triphenylphosphine unit. This effect confirms that the $3-E$ and $3-Z$ isomers appear as the minor and major forms, respectively (percentage of isomers reported in the Experimental Section) the signals for methine protons appeared as two doublets at $\delta=4.65 \mathrm{ppm}, J=16.7 \mathrm{~Hz}$ and $\delta=4.69 \mathrm{ppm}, J=18.7 \mathrm{~Hz}$, respectively for the $Z$ - and $E$ - isomers.

Selected ${ }^{1} \mathrm{H},{ }^{13} \mathrm{C}$, and ${ }^{31} \mathrm{P}$ NMR chemical shifts and coupling constants in the major (M) and minor $(\mathrm{m})$ geometrical isomers of compounds 3a-k are shown in Table 1. 
Table 1. Selected ${ }^{1} \mathrm{H},{ }^{13} \mathrm{C}$, and ${ }^{31} \mathrm{P}$ NMR chemical shifts ( $\delta$ in ppm) and coupling constants $(\mathrm{J}$ in $\mathrm{Hz}$ ) for $\mathrm{H}-2, \mathrm{OR}, \mathrm{CO}_{2} \mathrm{R}, \mathrm{C}-2$, and $\mathrm{C}-3$ in the major $(\mathrm{M})$ and minor (m) diastereoisomers of compounds 3a-k

\begin{tabular}{|c|c|c|c|c|c|c|c|}
\hline \multicolumn{3}{|c|}{${ }^{1} \mathrm{H}$ NMR spectroscopic data } & \multicolumn{3}{|c|}{${ }^{13} \mathrm{C}$ NMR data } & \multirow{2}{*}{$\frac{{ }^{31} \mathrm{P} \text { NMR }}{\mathrm{C}-3\left({ }^{1} J_{\mathrm{PC}}\right)}$} & \\
\hline Compd & Isomer $\%$ & $\mathrm{H}-2\left({ }^{3} J_{\mathrm{PH}}\right)$ & OR & $\mathrm{CO}_{2} \mathrm{R}$ & $\mathrm{C}-2\left({ }^{2} J_{\mathrm{PC}}\right)$ & & \\
\hline 3a & $M(75)$ & $4.65(16.7)$ & 3.06 & 3.87 & $63.20(14.1)$ & $40.55(124.7)$ & 23.53 \\
\hline 3a & $\mathrm{m}(25)$ & $4.69(18.7)$ & 3.42 & 3.78 & $65.48(15.1)$ & $42.26(133.9)$ & 23.91 \\
\hline $3 \mathbf{b}$ & $M(72)$ & $4.65(17.1)$ & 3.52 & 4.27 & $63.58(15.3)$ & $40.27(124.6)$ & 23.38 \\
\hline $3 \mathbf{b}$ & $\mathrm{m}(38)$ & $4.67(19.4)$ & 3.81 & 4.40 & $65.78(16.8)$ & $41.86(134.7)$ & 24.18 \\
\hline $3 c$ & M (70) & $4.63(16.6)$ & 3.06 & 3.87 & $63.92(15.7)$ & $40.47(124.7)$ & 23.59 \\
\hline 3c & $\mathrm{m}(30)$ & $4.67(18.7)$ & 3.37 & 3.81 & $66.46(14.9)$ & $42.46(133.3)$ & 24.15 \\
\hline 3d & M (64) & $5.56(10.0)$ & 3.17 & 3.81 & $62.31(15.2)$ & $40.72(125.6)$ & 22.77 \\
\hline 3d & m (36) & $5.03(9.1)$ & 3.57 & 3.82 & $65.12(15.8)$ & $42.23(133.8)$ & 23.78 \\
\hline 3e & M (68) & $5.61(17.2)$ & 3.72 & 4.26 & $62.36(14.0)$ & $40.80(127.2)$ & 22.69 \\
\hline 3e & m (32) & $5.11(19.4)$ & 3.32 & 4.33 & $61.98(14.7)$ & 41.97 (135.8) & 23.70 \\
\hline $3 f$ & M & $4.47(19.5)$ & 0.94 & 1.50 & $63.74(14.5)$ & $42.17(127.1)$ & 25.42 \\
\hline $3 g$ & $\mathrm{M}(62)$ & $4.67(17.2)$ & 3.49 & 4.28 & $62.15(14.8)$ & $40.65(125.3)$ & 23.48 \\
\hline $3 g$ & $\mathrm{~m}(38)$ & $4.69(19.4)$ & 3.71 & 4.39 & $65.90(15.9)$ & $42.06(133.6)$ & 24.56 \\
\hline $3 \mathbf{h}$ & M & $4.47(18.3)$ & 0.87 & 1.62 & $65.97(16.3)$ & $39.64(125.2)$ & 23.09 \\
\hline $3 \mathbf{i}$ & M (68) & $4.63(17.1)$ & 3.53 & 4.27 & $64.08(15.2)$ & 40.17 (124.6) & 23.39 \\
\hline $3 \mathbf{i}$ & m (32) & $4.65(19.4)$ & 3.81 & 4.43 & $66.45(17.9)$ & $42.26(133.7)$ & 24.29 \\
\hline $3 \mathbf{j}$ & M (72) & $4.62(16.5)$ & 3.07 & 3.88 & $63.81(15.0)$ & $40.27(125.0)$ & 23.51 \\
\hline $3 \mathbf{j}$ & m (28) & $4.68(17.4)$ & 3.42 & 3.80 & $66.35(14.8)$ & $42.06(133.7)$ & 23.91 \\
\hline $3 \mathbf{k}$ & M (70) & $4.63(17.0)$ & 3.57 & 3.78 & $63.79(14.3)$ & $40.20(124.4)$ & 23.38 \\
\hline $3 \mathbf{k}$ & $\mathrm{m}(30)$ & $4.64(19.5)$ & 4.27 & 4.40 & $65.90(16.1)$ & $41.80(133.6)$ & 24.18 \\
\hline
\end{tabular}

As can be seen, only one geometrical isomer was observed for the di-tert-butyl derivatives of 3 presumably because of the bulky tert-butyl groups. On the basis of the well-established chemistry of trivalent phosphorus nucleophiles, it is reasonable to assume that the phosphorus ylide 3 results from the initial addition of triphenylphosphine to the acetylenic ester and subsequent protonation of the 1:1adduct by the $\mathrm{NH}$ compound to form phosphoranes 3 (see Scheme 3). 


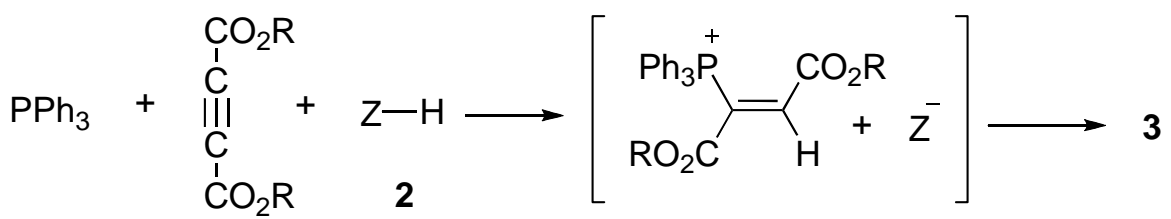

1

\section{Scheme 3}

In summary, we have prepared novel phosphorus ylides using a one-pot reaction between triphenylphosphine and dialkyl acetylenedicarboxylates in the presence of NH compounds such as 1-benzylidene-2-phenylhydrazine, 1-(3-chlorobenzylidene)-2-phenylhydrazine, 1-(2,4dinitrobenzylidene)-2-phenylhydrazine, 1-(4-nitrobenzylidene)-2-phenylhydrazine,1-(2fluorobenzylidene)-2-phenylhydrazine,1-(4-fluorobenzylidene)-2-phenylhydrazine or 1-(2,4dichlorobenzylidene)-2-phenylhydrazine. The present method has the advantage that not only is the reaction performed under neutral conditions, but also the substances can be mixed without any prior activation or modification. Phosphorus ylides 3a-k NH-acid containing may be considered potentially useful synthetic intermediates. The procedure described here may be an acceptable method for the preparation of phosphoranes with variable functionalities of phenylhydrazone.

\section{Experimental Section}

General Procedures. Melting points and IR spectra were measured on an Electrothermal 9100 apparatus and a Shimadzu IR-460 spectrometer, respectively. Also, the ${ }^{1} \mathrm{H},{ }^{13} \mathrm{C}$, and ${ }^{31} \mathrm{P}$ NMR spectra were obtained from a BRUKER DRX-500 AVANCE instrument with $\mathrm{CDCl}_{3}$ as a solvent at 500.1, 125.8, and 202.4 MHz, respectively. The mass spectra were recorded on a Shimadzu GCMS-QP 5050A mass spectrometer operating at an ionization potential of $70 \mathrm{eV}$. Triphenylphosphine, dialkyl acetylenedicarboxylates, were purchased from Fluka and phenylhydrazone derivatives prepared by the routine procedure. $^{32}$

General synthetic procedure, exemplified by dimethyl 2-(1-benzylidene-2-phenylhydrazine1-yl)-3-(triphenylphosphoranylidene) butanedioate (3a)

To a magnetically stirred solution of triphenylphosphine $(0.26 \mathrm{~g}, 1 \mathrm{mmol})$ and 1-benzylidene-2phenylhydrazine $(0.19 \mathrm{~g}, 1 \mathrm{mmol})$ in diethyl ether $(10 \mathrm{~mL})$, a mixture of dimethyl acetylenedicarboxylate $(0.14 \mathrm{~g}, 1 \mathrm{mmol})$ was added, dropwise, in diethyl ether $(4 \mathrm{~mL})$ at $-5{ }^{\circ} \mathrm{C}$ over $10 \mathrm{~min}$. After approximately $3 \mathrm{~h}$, the product was filtered off and recrystallized from diethyl ether.

Colorless crystals, yield $0.59 \mathrm{~g},(98 \%), \mathrm{mp} 139-141{ }^{\circ} \mathrm{C}, \mathrm{IR}(\mathrm{KBr})\left(\mathrm{v}_{\max }, \mathrm{cm}^{-1}\right): 1732,1646$ $(\mathrm{C}=\mathrm{O}) . \mathrm{MS}(\mathrm{m} / \mathrm{z}, \%): 600\left(\mathrm{M}^{+}, 2\right), 405$ (12), 277 (93), 262 (88), 196 (55), 183 (100), 108 (46), 92 
(40), 77 (100). Anal. Calcd for $\mathrm{C}_{37} \mathrm{H}_{33} \mathrm{~N}_{2} \mathrm{O}_{4} \mathrm{P}$ (600.64): C, 73.99; H, 5.54; N, 4.66\% Found: C, $74.08 ; \mathrm{H}, 5.46 ; \mathrm{N}, 4.59 \%$

Major rotamer (Z) (3a). (75\%): ${ }^{1} \mathrm{H}$ NMR (500.1 MHz, $\left.\mathrm{CDCl}_{3}\right): \delta 3.06$ and $3.87(6 \mathrm{H}, 2 \mathrm{~s}$, $\left.2 \mathrm{xOCH}_{3}\right), 4.65\left(1 \mathrm{H}, \mathrm{d},{ }^{3} \mathrm{~J}_{\mathrm{PH}}=16.7 \mathrm{~Hz}, \mathrm{P}=\mathrm{C}-\mathrm{CH}\right), 6.84(\mathrm{~s}, \mathrm{~N}=\mathrm{CH}), 7.16-7.69(25 \mathrm{H}, \mathrm{m}, \mathrm{Ar}-\mathrm{H})$. ${ }^{13} \mathrm{C} \mathrm{NMR}\left(125.8 \mathrm{MHz}, \mathrm{CDCl}_{3}\right): \delta 40.55\left(\mathrm{~d},{ }^{1} J_{\mathrm{PC}}=124.7 \mathrm{~Hz}, \mathrm{P}=\mathrm{C}\right), 49.25$ and $52.83\left(2 \mathrm{OCH}_{3}\right)$, $63.20\left(\mathrm{~d},{ }^{2} J_{\mathrm{PC}}=14.1 \mathrm{~Hz}, \mathrm{P}=\mathrm{C}-\mathrm{CH}\right), 126.01\left(\mathrm{~d},{ }^{1} J_{\mathrm{PC}}=91.8 \mathrm{~Hz}, \mathrm{C}_{\text {ipso }}\right), 128.63\left(\mathrm{~d},{ }^{3} J_{\mathrm{PC}}=12.0 \mathrm{~Hz}\right.$, $\left.\mathrm{C}_{\text {meta }}\right), 131.89\left(\mathrm{C}_{\text {para }}\right), 133.46\left(\mathrm{~d},{ }^{2} J_{\mathrm{PC}}=9.7 \mathrm{~Hz}, \mathrm{C}_{\text {ortho }}\right), 121.80,122.19,126.62,128.13,128.55$, 129.32, 137.23, $145.25\left(8 \mathrm{C}_{\mathrm{arom}}\right), 165.42(\mathrm{C}=\mathrm{N}), 169.90\left(\mathrm{~d},{ }^{2} J_{\mathrm{PC}}=12.6, \mathrm{C}=\mathrm{O}\right), 173.50\left(\mathrm{~d},{ }^{3} J_{\mathrm{PC}}=\right.$ $16.3 \mathrm{~Hz}, \mathrm{C}=\mathrm{O}) .{ }^{31} \mathrm{P}$ NMR $\left(202.4 \mathrm{MHz}, \mathrm{CDCl}_{3}\right): \delta 23.53\left(\mathrm{Ph}_{3} \mathrm{P}^{+}-\mathrm{C}\right)$.

Minor rotamer (E) (3a). (25\%): ${ }^{1} \mathrm{H} \mathrm{NMR}\left(500.1 \mathrm{MHz}, \mathrm{CDCl}_{3}\right): \delta 3.42$ and $3.78(6 \mathrm{H}, 2 \mathrm{~s}, 2$ $\left.\mathrm{OCH}_{3}\right), 4.69\left(1 \mathrm{H}, \mathrm{d},{ }^{3} J_{\mathrm{PH}}=18.7 \mathrm{~Hz}, \mathrm{P}=\mathrm{C}-\mathrm{CH}\right), 6.85(\mathrm{~s}, \mathrm{~N}=\mathrm{CH}), 7.16-7.69(25 \mathrm{H}, \mathrm{m}, 5 \mathrm{Ar}-\mathrm{H})$. ${ }^{13} \mathrm{C}$ NMR $\left(125.8 \mathrm{MHz} ; \mathrm{CDCl}_{3}\right): \delta 42.26\left(\mathrm{~d},{ }^{2} J_{\mathrm{PC}}=133.9 \mathrm{~Hz}, \mathrm{P}=\mathrm{C}\right), 50.05$ and $52.43\left(2 \mathrm{OCH}_{3}\right)$, $65.48\left(\mathrm{~d},{ }^{2} J_{\mathrm{PC}}=15.1 \mathrm{~Hz}, \mathrm{P}=\mathrm{C}-\mathrm{CH}\right), 126.22\left(\mathrm{~d},{ }^{1} J_{\mathrm{PC}}=97.1 \mathrm{~Hz}, \mathrm{C}_{\mathrm{ipso}}\right), 128.49\left(\mathrm{~d}, 3 J_{\mathrm{PC}}=11.8 \mathrm{~Hz}\right.$, $\left.\mathrm{C}_{\text {meta }}\right), 132.02\left(\mathrm{C}_{\text {para }}\right), 133.71\left(\mathrm{~d},{ }^{2} J_{\mathrm{PC}}=9.8 \mathrm{~Hz}, \mathrm{C}_{\text {ortho }}\right), 122.40,123.07,126.70,128.09,128.23$, 129.75, 137.11, 143.58 (8 $\left.\mathrm{C}_{\text {arom }}\right), 165.75(\mathrm{C}=\mathrm{N}), 169.83\left(\mathrm{~d},{ }^{2} J_{\mathrm{PC}}=17.7 \mathrm{~Hz}, \mathrm{C}=\mathrm{O}\right), 173.86(\mathrm{~d}$, $\left.{ }^{3} J_{\mathrm{PC}}=15.9 \mathrm{~Hz}, \mathrm{C}=\mathrm{O}\right) .{ }^{31} \mathrm{P} \mathrm{NMR}\left(202.4 \mathrm{MHz}, \mathrm{CDCl}_{3}\right): \delta 23.91\left(\mathrm{Ph}_{3} \mathrm{P}^{+}-\mathrm{C}\right)$.

Diethyl 2-(1-benzylidene-2-phenylhydrazine-1-yl)-3-(triphenylphosphoranylidene) butanedioate (3b). Colorless crystals, yield $0.61 \mathrm{~g},(97 \%), \mathrm{mp} 135-137^{\circ} \mathrm{C}$, IR $(\mathrm{KBr})\left(\mathrm{v}_{\max }, \mathrm{cm}^{-1}\right): 1726,1647(\mathrm{C}=\mathrm{O}) . \mathrm{MS}(\mathrm{m} / \mathrm{z}$, \%): $628\left(\mathrm{M}^{+}, 1\right), 433$ (16), 277 (100), 262 (71), 196 (97), 183 (83), 108 (35), 92 (72), 77 (94). Anal. Calcd for $\mathrm{C}_{39} \mathrm{H}_{37} \mathrm{~N}_{2} \mathrm{O}_{4} \mathrm{P}$ (628.69): C, 74.51; H, 5.93; N, 4.46\% Found: C, 74.58; H, 5.85; N, 4.49\%

Major rotamer (Z) (3b). (72\%), ${ }^{1} \mathrm{H} \mathrm{NMR}\left(500.1 \mathrm{MHz}, \mathrm{CDCl}_{3}\right): 0.39$ and $1.29\left(6 \mathrm{H}, 2 \mathrm{t},{ }^{3} J_{\mathrm{HH}}=\right.$ $\left.7.0 \mathrm{~Hz}, 2 \mathrm{CH}_{3}\right), 3.52$ and $4.27\left(4 \mathrm{H}, 2 \mathrm{~m}, 2 \mathrm{OCH}_{2}\right), 4.65\left(1 \mathrm{H}, \mathrm{d},{ }^{3} J_{\mathrm{PH}}=17.1 \mathrm{~Hz}, \mathrm{P}=\mathrm{C}-\mathrm{CH}\right), 6.87$ $(\mathrm{s}, \mathrm{N}=\mathrm{CH}), 6.82-7.79(25 \mathrm{H}, \mathrm{m}, 5 \mathrm{Ar}-\mathrm{H}) .{ }^{13} \mathrm{C} \mathrm{NMR}\left(125.8 \mathrm{MHz}, \mathrm{CDCl}_{3}\right): \delta 14.01$ and $14.34(2$ $\left.\mathrm{CH}_{3}\right), 40.27\left(\mathrm{~d},{ }^{1} J_{\mathrm{PC}}=124.6 \mathrm{~Hz}, \mathrm{P}=\mathrm{C}\right), 57.69$ and $61.43\left(2 \mathrm{OCH}_{2}\right), 63.58\left(\mathrm{~d},{ }^{2} J_{\mathrm{PC}}=15.3 \mathrm{~Hz}\right.$, $\mathrm{P}=\mathrm{C}-\mathrm{CH}), 126.34\left(\mathrm{~d},{ }^{1} J_{\mathrm{PC}}=91.7 \mathrm{~Hz}, \mathrm{C}_{\mathrm{ipso}}\right), 128.52\left(\mathrm{~d},{ }^{3} J_{\mathrm{PC}}=12.2 \mathrm{~Hz}, \mathrm{C}_{\text {meta }}\right), 131.80\left(\mathrm{C}_{\text {para }}\right)$, $133.57\left(\mathrm{~d},{ }^{2} J_{\mathrm{PC}}=9.8 \mathrm{~Hz}, \mathrm{C}_{\text {ortho }}\right), 119.65,121.98,126.11,128.11,129.21,129.82,137.39,145.47$ $\left(8 \mathrm{C}_{\text {arom }}\right), 165.03(\mathrm{C}=\mathrm{N}), 168.53\left(\mathrm{~d},{ }^{2} J_{\mathrm{PC}}=12.6 \mathrm{~Hz}, \mathrm{C}=\mathrm{O}\right), 172.81\left(\mathrm{~d},{ }^{3} J_{\mathrm{PC}}=16.1 \mathrm{~Hz}, \mathrm{C}=\mathrm{O}\right) .{ }^{31} \mathrm{P}$ NMR (202.4 MHz, $\left.\mathrm{CDCl}_{3}\right): \delta 23.38\left(\mathrm{Ph}_{3} \mathrm{P}^{+}-\mathrm{C}\right)$.

Minor rotamer (E) (3b). (28\%), ${ }^{1} \mathrm{H}$ NMR (500.1 MHz, $\left.\mathrm{CDCl}_{3}\right): \delta 1.15$ and $1.34\left(6 \mathrm{H}, 2 \mathrm{t},{ }^{3} J_{\mathrm{HH}}=\right.$ $\left.7.0 \mathrm{~Hz}, 2 \mathrm{CH}_{3}\right), 3.81$ and $4.40\left(4 \mathrm{H}, 2 \mathrm{~m}, 2 \mathrm{OCH}_{2}\right), 4.67\left(1 \mathrm{H}, \mathrm{d},{ }^{3} J_{\mathrm{PH}}=19.4 \mathrm{~Hz}, \mathrm{P}=\mathrm{C}-\mathrm{CH}\right), 6.86$ $(\mathrm{s}, \mathrm{N}=\mathrm{CH}), 6.82-7.79(25 \mathrm{H}, \mathrm{m}, 5 \mathrm{Ar}-H) .{ }^{13} \mathrm{C} \mathrm{NMR}\left(125.8 \mathrm{MHz} ; \mathrm{CDCl}_{3}\right): \delta 14.12$ and $14.78(2$ $\left.\mathrm{CH}_{3}\right), 41.86\left(\mathrm{~d},{ }^{1} J_{\mathrm{PC}}=134.7 \mathrm{~Hz}, \mathrm{P}=\mathrm{C}\right), 58.25$ and $60.88\left(2 \mathrm{OCH}_{2}\right), 65.78\left(\mathrm{~d},{ }^{2} J_{\mathrm{PC}}=16.8 \mathrm{~Hz}\right.$, $\mathrm{P}=\mathrm{C}-\mathrm{CH}), 126.14\left(\mathrm{~d},{ }^{1} J_{\mathrm{PC}}=92.2 \mathrm{~Hz}, \mathrm{C}_{\mathrm{ipso}}\right), 128.59\left(\mathrm{~d},{ }^{3} J_{\mathrm{PC}}=12.0 \mathrm{~Hz}, \mathrm{C}_{\text {meta }}\right), 132.04\left(\mathrm{C}_{\text {para }}\right)$, $133.71\left(\mathrm{~d},{ }^{2} J_{\mathrm{PC}}=12.3 \mathrm{~Hz}, \mathrm{C}_{\text {ortho }}\right), 119.78,122.30,126.57,128.01,129.41,129.87,137.12,144.80$ $\left(8 \mathrm{C}_{\text {arom }}\right), 165.23(\mathrm{C}=\mathrm{N}), 169.67\left(\mathrm{~d},{ }^{2} J_{\mathrm{PC}}=15.1 \mathrm{~Hz}, \mathrm{C}=\mathrm{O}\right), 173.28\left(\mathrm{~d},{ }^{3} J_{\mathrm{PC}}=16.1 \mathrm{~Hz}, \mathrm{C}=\mathrm{O}\right) .{ }^{31} \mathrm{P}$ NMR (202.4 MHz, $\left.\mathrm{CDCl}_{3}\right): \delta 24.18\left(\mathrm{Ph}_{3} \mathrm{P}^{+}-\mathrm{C}\right)$.

Dimethyl 2-(1-(3-chlorobenzylidene)-2-phenylhydrazine-1-yl)-3-(triphenylphosphoranylidene)butandioate (3c). Colorless crystals, yield $0.61 \mathrm{~g},(97 \%), \mathrm{mp} 139-141^{\circ} \mathrm{C}, \mathrm{IR}(\mathrm{KBr})\left(\mathrm{v}_{\max }\right.$, $\left.\mathrm{cm}^{-1}\right): 1724,1647(\mathrm{C}=\mathrm{O})$. MS (m/z, \%): $635\left(\mathrm{M}^{+}, 1\right), 405$ (4), 277 (83), 262 (100), 230 (38), 183 
(88), 108 (36), 92 (46), 77 (77). Anal. Calcd for $\mathrm{C}_{37} \mathrm{H}_{32} \mathrm{ClN}_{2} \mathrm{O}_{4} \mathrm{P}$ (635.09): C, 69.97; H, 5.08; N, 4.41\% Found: C, 70.22; H, 4.95; N, 4.37\%

Major isomer (Z) (3c). (70\%), ${ }^{1} \mathrm{H} \mathrm{NMR}\left(500.1 \mathrm{MHz}, \mathrm{CDCl}_{3}\right): \delta 3.06$ and $3.87(6 \mathrm{H}, 2 \mathrm{~s}, 2$ $\left.\mathrm{OCH}_{3}\right), 4.63\left(1 \mathrm{H}, \mathrm{d},{ }^{3} \mathrm{~J}_{\mathrm{PH}}=16.6 \mathrm{~Hz}, \mathrm{P}=\mathrm{C}-\mathrm{CH}\right), 6.79(\mathrm{~s}, \mathrm{~N}=\mathrm{CH}), 6.74-8.05(24 \mathrm{H}, \mathrm{m}, 5 \mathrm{Ar}-\mathrm{H})$. ${ }^{13} \mathrm{C} \mathrm{NMR}\left(125.8 \mathrm{MHz}, \mathrm{CDCl}_{3}\right): \delta 40.47\left(\mathrm{~d},{ }^{1} J_{\mathrm{PC}}=124.7 \mathrm{~Hz}, \mathrm{P}=\mathrm{C}\right), 49.21$ and $52.81\left(2 \mathrm{OCH}_{3}\right)$, $63.92\left(\mathrm{~d},{ }^{2} J_{\mathrm{PC}}=15.7 \mathrm{~Hz}, \mathrm{P}=\mathrm{C}-\mathrm{CH}\right), 126.09\left(\mathrm{~d},{ }^{1} J_{\mathrm{PC}}=91.7 \mathrm{~Hz}, \mathrm{C}_{\mathrm{ipso}}\right), 128.52\left(\mathrm{~d},{ }^{3} J_{\mathrm{PC}}=12.0 \mathrm{~Hz}\right.$, $\left.\mathrm{C}_{\text {meta }}\right), 132.02\left(\mathrm{C}_{\text {para }}\right), 133.47\left(\mathrm{~d},{ }^{2} J_{\mathrm{PC}}=9.6 \mathrm{~Hz}, \mathrm{C}_{\text {ortho }}\right), 122.26,124.00,126.61,128.18,129.34$, $129.75,134.55,144.83\left(8 \mathrm{C}_{\mathrm{arom}}\right), 165.40(\mathrm{C}=\mathrm{N}), 168.98\left(\mathrm{~d},{ }^{2} J_{\mathrm{PC}}=12.6 \mathrm{~Hz}, \mathrm{C}=\mathrm{O}\right), 173.21\left(\mathrm{~d},{ }^{3} J_{\mathrm{PC}}\right.$ $=16.1 \mathrm{~Hz}, \mathrm{C}=\mathrm{O}) .{ }^{31} \mathrm{P}$ NMR $\left(202.4 \mathrm{MHz}, \mathrm{CDCl}_{3}\right): \delta 23.59\left(\mathrm{Ph}_{3} \mathrm{P}^{+}-\mathrm{C}\right)$.

Minor isomer (E) (3c). (30\%), ${ }^{1} \mathrm{H} \mathrm{NMR}\left(500.1 \mathrm{MHz}, \mathrm{CDCl}_{3}\right): \delta 3.37$ and $3.81(6 \mathrm{H}, 2 \mathrm{~s}, 2$ $\left.\mathrm{OCH}_{3}\right), 4.67\left(1 \mathrm{H},{ }^{3} \mathrm{~J}_{\mathrm{PH}}=18.7 \mathrm{~Hz}, \mathrm{P}=\mathrm{C}-\mathrm{CH}\right), 6.78(\mathrm{~s}, \mathrm{~N}=\mathrm{CH}), 6.74-8.05(24 \mathrm{H}, \mathrm{m}, 5 \mathrm{Ar}-\mathrm{H}) .{ }^{13} \mathrm{C}$ NMR (125.8 MHz, $\left.\mathrm{CDCl}_{3}\right): \delta 42.46\left(\mathrm{~d},{ }^{1} J_{\mathrm{PC}}=133.3 \mathrm{~Hz}, \mathrm{P}=\mathrm{C}\right), 49.94$ and $52.36\left(2 \mathrm{OCH}_{3}\right), 66.46$ $\left(\mathrm{d},{ }^{2} J_{\mathrm{PC}}=14.9 \mathrm{~Hz}, \mathrm{P}=\mathrm{C}-\mathrm{CH}\right), 126.34\left(\mathrm{~d},{ }^{1} J_{\mathrm{PC}}=92.0, \mathrm{C}_{\mathrm{ipso}}\right), 128.64\left(\mathrm{~d},{ }^{3} J_{\mathrm{PC}}=12.3 \mathrm{~Hz}, \mathrm{C}_{\text {meta }}\right)$, $132.15\left(\mathrm{C}_{\mathrm{para}}\right), 133.74\left(\mathrm{~d},{ }^{2} J_{\mathrm{PC}}=9.7 \mathrm{~Hz}, \mathrm{C}_{\text {ortho }}\right), 120.08,123.83,126.36,127.93,129.49,129.23$, 134.18, $144.47\left(8 \mathrm{C}_{\text {arom }}\right), 165.40(\mathrm{C}=\mathrm{N}), 169.82\left(\mathrm{~d},{ }^{2} J_{\mathrm{PC}}=17.7 \mathrm{~Hz}, \mathrm{C}=\mathrm{O}\right), 173.81\left(\mathrm{~d},{ }^{3} J_{\mathrm{PC}}=15.6\right.$ $\mathrm{Hz}, \mathrm{C}=\mathrm{O}) .{ }^{31} \mathrm{P}$ NMR $\left(202.4 \mathrm{MHz}, \mathrm{CDCl}_{3}\right): \delta 24.15\left(\mathrm{Ph}_{3} \mathrm{P}^{+}-\mathrm{C}\right)$.

Dimethyl 2-(1-(2,4-dinitrobenzylidene)-2-phenylhydrazine-1-yl)-3(triphenylphosphoranylidene)butandioate (3d). Colorless crystals, yield $0.68 \mathrm{~g},(98 \%), \mathrm{mp}$ 140-142 C, IR (KBr) $\left(v_{\max }, \mathrm{cm}^{-1}\right): 1734,1618(\mathrm{C}=\mathrm{O}) . \mathrm{MS}(\mathrm{m} / \mathrm{z}, \%): 690\left(\mathrm{M}^{+}, 3\right), 405(7), 277$ (100), 262 (46), 183 (76), 108 (24), 77 (57). Anal. Calcd for $\mathrm{C}_{37} \mathrm{H}_{31} \mathrm{~N}_{4} \mathrm{O}_{8} \mathrm{P}$ (690.63): C, 64.35; H, $4.52 ; \mathrm{N}, 8.11 \%$ Found: C, 64.42; H, 4.53; N, 8.08\%

Major isomer (Z)(3d). (64\%), ${ }^{1} \mathrm{H}$ NMR (500.1 MHz, $\left.\mathrm{CDCl}_{3}\right): \delta 3.17$ and $3.81(6 \mathrm{H}, 2 \mathrm{~s}, 2$ $\left.\mathrm{OCH}_{3}\right), 5.56\left(1 \mathrm{H}, \mathrm{d},{ }^{3} \mathrm{~J}_{\mathrm{PH}}=10.0 \mathrm{~Hz}, \mathrm{P}=\mathrm{C}-\mathrm{CH}\right), 7.36(\mathrm{~s}, \mathrm{~N}=\mathrm{CH}), 7.38-9.99(23 \mathrm{H}, \mathrm{m}, 5 \mathrm{Ar}-\mathrm{H})$. ${ }^{13} \mathrm{C} \mathrm{NMR}\left(125.8 \mathrm{MHz}, \mathrm{CDCl}_{3}\right): \delta 40.72\left(\mathrm{~d},{ }^{1} J_{\mathrm{PC}}=125.6 \mathrm{~Hz}, \mathrm{P}=\mathrm{C}\right), 49.34$ and $52.36\left(2 \mathrm{OCH}_{3}\right)$, $62.31\left(\mathrm{~d},{ }^{2} J_{\mathrm{PC}}=15.2 \mathrm{~Hz}, \mathrm{P}=\mathrm{C}-\mathrm{CH}\right), 126.78\left(\mathrm{~d},{ }^{1} J_{\mathrm{PC}}=92.0 \mathrm{~Hz}, \mathrm{C}_{\mathrm{ipso}}\right), 128.89\left(\mathrm{~d},{ }^{3} J_{\mathrm{PC}}=12.1 \mathrm{~Hz}\right.$, $\left.\mathrm{C}_{\text {meta }}\right), 132.01\left(\mathrm{C}_{\text {para }}\right), 133.58\left(\mathrm{~d},{ }^{2} J_{\mathrm{PC}}=9.8 \mathrm{~Hz}, \mathrm{C}_{\text {ortho }}\right), 117.53,123.81,128.59,129.42,132.40$, 135.98, 144.13, $149.42\left(8 \mathrm{C}_{\text {arom }}\right), 165.11(\mathrm{C}=\mathrm{N}), 169.80\left(\mathrm{~d},{ }^{2} J_{\mathrm{PC}}=12.7 \mathrm{~Hz}, \mathrm{C}=\mathrm{O}\right), 173.98\left(\mathrm{~d},{ }^{3} J_{\mathrm{PC}}\right.$ $=9.5 \mathrm{~Hz}, \mathrm{C}=\mathrm{O}) .{ }^{31} \mathrm{P}$ NMR $\left(202.4 \mathrm{MHz}, \mathrm{CDCl}_{3}\right): \delta 22.77\left(\mathrm{Ph}_{3} \mathrm{P}^{+}-\mathrm{C}\right)$.

Minor isomer (E) (3d). (36\%), ${ }^{1} \mathrm{H} \mathrm{NMR}\left(500.1 \mathrm{MHz}, \mathrm{CDCl}_{3}\right): \delta 3.57$ and $3.82(6 \mathrm{H}, 2 \mathrm{~s}, 2$ $\left.\mathrm{OCH}_{3}\right), 5.03\left(1 \mathrm{H}, \mathrm{d},{ }^{3} J_{\mathrm{PH}}=9.1 \mathrm{~Hz}, \mathrm{P}=\mathrm{C}-\mathrm{CH}\right), 7.33(\mathrm{~s}, \mathrm{~N}=\mathrm{CH}), 7.38-9.99(23 \mathrm{H}, \mathrm{m}, 5 \mathrm{Ar}-\mathrm{H}) .{ }^{13} \mathrm{C}$ NMR (125.8 MHz, $\left.\mathrm{CDCl}_{3}\right): \delta 42.23\left(\mathrm{~d},{ }^{1} \mathrm{~J}_{\mathrm{PC}}=133.8 \mathrm{~Hz}, \mathrm{P}=\mathrm{C}\right), 50.06$ and $52.28\left(2 \mathrm{OCH}_{3}\right), 65.12$ $\left(\mathrm{d},{ }^{2} J_{\mathrm{PC}}=15.8 \mathrm{~Hz}, \mathrm{P}=\mathrm{C}-\mathrm{CH}\right), 126.93\left(\mathrm{~d},{ }^{1} J_{\mathrm{PC}}=94.0 \mathrm{~Hz}, \mathrm{C}_{\mathrm{ipso}}\right), 128.85\left(\mathrm{~d},{ }^{3} J_{\mathrm{PC}}=12.3 \mathrm{~Hz}, \mathrm{C}_{\text {meta }}\right)$, $132.14\left(\mathrm{C}_{\text {para }}\right), 133.65\left(\mathrm{~d},{ }^{2} J_{\mathrm{PC}}=9.8 \mathrm{~Hz}, \mathrm{C}_{\text {ortho }}\right), 115.44,120.10,122.43,129.38,132.37,135.90$, $143.79,149.38\left(8 \mathrm{C}_{\text {arom }}\right), 165.11(\mathrm{C}=\mathrm{N}), 170.05\left(\mathrm{~d},{ }^{2} J_{\mathrm{PC}}=15.7 \mathrm{~Hz}, \mathrm{C}=\mathrm{O}\right), 174.51\left(\mathrm{~d},{ }^{3} J_{\mathrm{PC}}=12.2\right.$ $\mathrm{Hz}, \mathrm{C}=\mathrm{O}) .{ }^{31} \mathrm{P}$ NMR $\left(202.4 \mathrm{MHz}, \mathrm{CDCl}_{3}\right): \delta 23.78\left(\mathrm{Ph}_{3} \mathrm{P}^{+}-\mathrm{C}\right)$.

Diethyl 2-(1-(2,4-dinitrobenzylidene)-2-phenylhydrazine-1-yl)-3-(triphenylphosphoranylidene)butandioate (3e). Colorless crystals, yield $0.70 \mathrm{~g}$, (97\%), mp 136-138 $\mathrm{C}$, IR (KBr) $\left(v_{\max }, \mathrm{cm}^{-1}\right): 1725,1610(\mathrm{C}=\mathrm{O}) . \mathrm{MS}(\mathrm{m} / \mathrm{z}, \%): 718\left(\mathrm{M}^{+}, 5\right), 433$ (22), $277(100), 262(47), 183$ (38), 77 (60). Anal. Calcd for $\mathrm{C}_{39} \mathrm{H}_{35} \mathrm{~N}_{4} \mathrm{O}_{8} \mathrm{P}$ (718.69): C, 65.18; H, 4.91; N, 7.80\% Found: C, $65.26 ; \mathrm{H}, 4.92 ; \mathrm{N}, 7.74 \%$ 
Major isomer (Z) (3e). (68\%), ${ }^{1} \mathrm{H}$ NMR (500.1 MHz, $\left.\mathrm{CDCl}_{3}\right): \delta 0.47\left(3 \mathrm{H}, 2 \mathrm{t},{ }^{3} J_{\mathrm{HH}}=7.0 \mathrm{~Hz}\right.$, $\left.\mathrm{CH}_{3}\right), 1.32\left(3 \mathrm{H}, \mathrm{t},{ }^{3} J_{\mathrm{HH}}=7.1 \mathrm{~Hz}, \mathrm{CH}_{3}\right), 3.72$ and $4.26\left(4 \mathrm{H}, 2 \mathrm{~m}, 2 \mathrm{OCH}_{2}\right), 5.61\left(1 \mathrm{H}, \mathrm{d},{ }^{3} J_{\mathrm{PH}}=\right.$ 17.2 Hz, P=C-CH), $7.39(\mathrm{~s}, \mathrm{~N}=\mathrm{CH}), 7.48-10.01(23 \mathrm{H}, \mathrm{m}, 5 \mathrm{Ar}-\mathrm{H}) .{ }^{13} \mathrm{C}$ NMR $\left(125.8 \mathrm{~Hz}, \mathrm{CDCl}_{3}\right)$ : $\delta 13.87$ and $14.22\left(2 \mathrm{CH}_{3}\right), 40.80\left(\mathrm{~d},{ }^{1} J_{\mathrm{PC}}=127.2 \mathrm{~Hz}, \mathrm{P}=\mathrm{C}\right), 57.92$ and $61.35\left(2 \mathrm{OCH}_{2}\right), 62.36(\mathrm{~d}$, $\left.{ }^{2} J_{\mathrm{PC}}=14.0 \mathrm{~Hz}, \mathrm{P}=\mathrm{C}-\mathrm{CH}\right), 126.44\left(\mathrm{~d},{ }^{1} J_{\mathrm{PC}}=92.3 \mathrm{~Hz}, \mathrm{C}_{\mathrm{ipso}}\right), 128.78\left(\mathrm{~d},{ }^{3} J_{\mathrm{PC}}=12.1 \mathrm{~Hz}, \mathrm{C}_{\text {meta }}\right)$, $132.37\left(\mathrm{C}_{\text {para }}\right), 133.63\left(\mathrm{~d},{ }^{2} J_{\mathrm{pc}}=9.8 \mathrm{~Hz}, \mathrm{C}_{\text {ortho }}\right), 115.52,123.92,126.14,128.79,132.37,135.79$, 145.11, $149.47\left(8 \mathrm{C}_{\text {arom }}\right), 165.08(\mathrm{C}=\mathrm{N}), 169.63\left(\mathrm{~d},{ }^{2} J_{\mathrm{PC}}=12.5 \mathrm{~Hz}, \mathrm{C}=\mathrm{O}\right), 173.82\left(\mathrm{~d},{ }^{3} J_{\mathrm{PC}}=9.0\right.$ $\mathrm{Hz}, \mathrm{C}=\mathrm{O}) .{ }^{31} \mathrm{P}$ NMR $\left(202.4 \mathrm{MHz}, \mathrm{CDCl}_{3}\right): \delta 22.69\left(\mathrm{Ph}_{3} \mathrm{P}^{+}-\mathrm{C}\right)$.

Minor isomer (E) (3e). (32\%), ${ }^{1} \mathrm{H}$ NMR (500.1 MHz, $\left.\mathrm{CDCl}_{3}\right): \delta 1.15\left(3 \mathrm{H}, \mathrm{t},{ }^{3} \mathrm{~J}_{\mathrm{HH}}=7.0 \mathrm{~Hz}\right.$, $\left.\mathrm{CH}_{3}\right), 1.67\left(3 \mathrm{H}, \mathrm{t},{ }^{3} J_{\mathrm{HH}}=7.2 \mathrm{~Hz}, \mathrm{CH}_{3}\right), 3.32$ and $4.33\left(4 \mathrm{H}, 2 \mathrm{~m}, 2 \mathrm{OCH}_{2}\right), 5.11\left(1 \mathrm{H}, \mathrm{d},{ }^{3} J_{\mathrm{PH}}=\right.$ $19.4 \mathrm{~Hz}, \mathrm{P}=\mathrm{C}-\mathrm{CH}), 7.36(\mathrm{~s}, \mathrm{~N}=\mathrm{CH}), 7.48-10.01(23 \mathrm{H}, \mathrm{m}, 5 \mathrm{Ar}-\mathrm{H}) .{ }^{13} \mathrm{C} \mathrm{NMR}(125.8 \mathrm{MHz}$, $\left.\mathrm{CDCl}_{3}\right): \delta 14.01$ and $14.99\left(2 \mathrm{CH}_{3}\right), 41.97\left(\mathrm{~d},{ }^{1} J_{\mathrm{PC}}=135.8 \mathrm{~Hz}, \mathrm{P}=\mathrm{C}\right), 58.49$ and $61.40\left(2 \mathrm{OCH}_{2}\right)$, $61.98\left(\mathrm{~d},{ }^{2} J_{\mathrm{PC}}=14.7 \mathrm{~Hz}, \mathrm{P}=\mathrm{C}-\mathrm{CH}\right), 125.92\left(\mathrm{~d},{ }^{1} J_{\mathrm{PC}}=92.3 \mathrm{~Hz}, \mathrm{C}_{\mathrm{ipso}}\right), 128.51\left(\mathrm{~d},{ }^{3} J_{\mathrm{PC}}=12.0 \mathrm{~Hz}\right.$, $\left.\mathrm{C}_{\text {meta }}\right), 132.02\left(\mathrm{C}_{\text {para }}\right), 133.27\left(\mathrm{~d},{ }^{2} J_{\mathrm{PC}}=9.8 \mathrm{~Hz}, \mathrm{C}_{\text {ortho }}\right), 115.37,123.83,123.07,128.95,133.41$, 135.88, 145.07, $149.45\left(8 \mathrm{C}_{\text {arom }}\right), 165.08(\mathrm{C}=\mathrm{N}), 169.78\left(\mathrm{~d},{ }^{2} J_{\mathrm{PC}}=16.0 \mathrm{~Hz}, \mathrm{C}=\mathrm{O}\right), 174.46\left(\mathrm{~d},{ }^{3} J_{\mathrm{PC}}\right.$ $=9.2 \mathrm{~Hz}, \mathrm{C}=\mathrm{O}) .{ }^{31} \mathrm{P}$ NMR $\left(202.4 \mathrm{MHz}, \mathrm{CDCl}_{3}\right): \delta 23.70\left(\mathrm{Ph}_{3} \mathrm{P}^{+}-\mathrm{C}\right)$.

Di-tert-butyl 2-(1-(2,4-dinitrobenzylidene)-2-phenylhydrazine-1-yl)-3-(triphenylphosphoranylidene)butandioate (3f). Colorless crystals, yield 0.76 g, (98\%), mp 189-191 C, IR (KBr) $\left(v_{\max }, \mathrm{cm}^{-1}\right): 1733,1635(\mathrm{C}=\mathrm{O})$. MS (m/z, \%): $774\left(\mathrm{M}^{+}, 3\right), 486$ (15), 262 (100), 77 (48). Anal. Calcd for $\mathrm{C}_{43} \mathrm{H}_{43} \mathrm{~N}_{4} \mathrm{O}_{8} \mathrm{P}$ (774.79): C, 66.66; H, 5.59; N, 7.23. Found: C, 66.69; H, 5.59; N, 7.28. ${ }^{1} \mathrm{H} \mathrm{NMR}\left(500.1 \mathrm{MHz}, \mathrm{CDCl}_{3}\right): \delta 0.94$ and $1.50\left(18 \mathrm{H}, 2 \mathrm{~s}, 2 \mathrm{CMe}_{3}\right), 4.47\left(1 \mathrm{H}, \mathrm{d},{ }^{3} J_{\mathrm{PH}}=19.5 \mathrm{~Hz}\right.$, $\mathrm{P}=\mathrm{C}-\mathrm{CH}), 7.11(\mathrm{~s}, \mathrm{~N}=\mathrm{CH}), 6.68-10.19(23 \mathrm{H}, \mathrm{m}, 5 \mathrm{Ar}-\mathrm{H}) .{ }^{13} \mathrm{C} \mathrm{NMR}\left(125.8 \mathrm{MHz}, \mathrm{CDCl}_{3}\right): \delta$ 27.98 and $28.26\left(2 \mathrm{CMe}_{3}\right), 42.17\left(\mathrm{~d},{ }^{1} J_{\mathrm{PC}}=127.1 \mathrm{~Hz}, \mathrm{P}=\mathrm{C}\right), 63.74\left(\mathrm{~d},{ }^{2} J_{\mathrm{PC}}=14.5 \mathrm{~Hz}, \mathrm{P}=\mathrm{C}-\mathrm{CH}\right)$, $127.08\left(\mathrm{~d},{ }^{1} J_{\mathrm{PC}}=92.2 \mathrm{~Hz}, \mathrm{C}_{\text {ipso }}\right), 128.48\left(\mathrm{~d},{ }^{3} J_{\mathrm{PC}}=12.1 \mathrm{~Hz}, \mathrm{C}_{\text {meta }}\right), 131.89\left(\mathrm{C}_{\mathrm{para}}\right), 133.71\left(\mathrm{~d},{ }^{2} J_{\mathrm{PC}}\right.$ $\left.=9.7 \mathrm{~Hz}, \mathrm{C}_{\text {ortho}}\right), 113.09,120.91,123.21,123.76,128.58,129.30,144.72,145.58$ (8 $\mathrm{C}_{\text {arom }}$ ), $164.43(\mathrm{C}=\mathrm{N}), 168.73\left(\mathrm{~d},{ }^{2} J_{\mathrm{PC}}=12.3 \mathrm{~Hz}, \mathrm{C}=\mathrm{O}\right), 172.16\left(\mathrm{~d},{ }^{3} J_{\mathrm{PC}}=9.9 \mathrm{~Hz}, \mathrm{C}=\mathrm{O}\right) .{ }^{31} \mathrm{P}$ NMR $\left(202.4 \mathrm{MHz}, \mathrm{CDCl}_{3}\right): \delta 25.42\left(\mathrm{Ph}_{3} \mathrm{P}^{+}-\mathrm{C}\right)$.

Diethyl 2-(1-(4-nitrobenzylidene)-2-phenylhydrazine-1-yl)-3-(triphenylphosphoranylidene)butandioate (3g). Colorless crystals, yield $0.65 \mathrm{~g},(98 \%), \mathrm{mp} 105-107^{\circ} \mathrm{C}, \mathrm{IR}(\mathrm{KBr})\left(\mathrm{v}_{\max }\right.$, $\left.\mathrm{cm}^{-1}\right)$ : 1718, $1639(\mathrm{C}=\mathrm{O})$. MS (m/z, \%): $637\left(\mathrm{M}^{+}, 1\right), 433$ (7), 365 (18), 337 (8), 277 (43), 262 (66), 183 (71), 108 (33), 92 (9), 77 (100). Anal. Calcd for $\mathrm{C}_{39} \mathrm{H}_{36} \mathrm{~N}_{3} \mathrm{O}_{6} \mathrm{P}$ (637.69): C, 69.53; H, 5.39; N, 6.24\% Found: C, 69.58; H, 5.31; N, 6.20\%

Major isomer (Z) (3g). (62\%), ${ }^{1} \mathrm{H}$ NMR $\left(500.1 \mathrm{MHz}, \mathrm{CDCl}_{3}\right): \delta 0.39\left(3 \mathrm{H}, \mathrm{t},{ }^{3} J_{\mathrm{HH}}=7.0 \mathrm{~Hz}\right.$, $\left.\mathrm{CH}_{3}\right), 1.21\left(3 \mathrm{H}, \mathrm{t},{ }^{3} \mathrm{~J}_{\mathrm{HH}}=6.9 \mathrm{~Hz}, \mathrm{CH}_{3}\right), 3.49$ and $4.28\left(4 \mathrm{H}, 2 \mathrm{~m}, 2 \mathrm{OCH}_{2}\right), 4.67\left(1 \mathrm{H},{ }^{3} J_{\mathrm{PH}}=17.2\right.$ $\mathrm{Hz}, \mathrm{P}=\mathrm{C}-\mathrm{CH}), 6.86(\mathrm{~s}, \mathrm{~N}=\mathrm{CH}), 6.74-8.67(24 \mathrm{H}, \mathrm{m}, 5 \mathrm{Ar}-\mathrm{H}) .{ }^{13} \mathrm{C} \mathrm{NMR}\left(500.1 \mathrm{MHz}, \mathrm{CDCl}_{3}\right): \delta$ 14.11 and $15.29\left(2 \mathrm{CH}_{3}\right), 40.65\left(\mathrm{~d},{ }^{1} J_{\mathrm{PC}}=125.3 \mathrm{~Hz}, \mathrm{P}=\mathrm{C}\right), 57.51$ and $61.34\left(2 \mathrm{OCH}_{2}\right), 62.15(\mathrm{~d}$, $\left.{ }^{2} J_{\mathrm{PC}}=14.8 \mathrm{~Hz}, \mathrm{P}=\mathrm{C}-\mathrm{CH}\right), 126.30\left(\mathrm{~d},{ }^{1} J_{\mathrm{PC}}=92.3 \mathrm{~Hz}, \mathrm{C}_{\mathrm{ipso}}\right), 128.51\left(\mathrm{~d},{ }^{3} J_{\mathrm{PC}}=12.0 \mathrm{~Hz}, \mathrm{C}_{\text {meta }}\right)$, $132.02\left(\mathrm{C}_{\mathrm{para}}\right), 133.56\left(\mathrm{~d},{ }^{2} J_{\mathrm{PC}}=11.5 \mathrm{~Hz}, \mathrm{C}_{\text {ortho }}\right), 113.08,120.90,123.36,124.05,128.28,129.37$, 144.31, $146.73\left(8 \mathrm{C}_{\text {arom }}\right), 165.02(\mathrm{C}=\mathrm{N}), 168.81\left(\mathrm{~d},{ }^{2} J_{\mathrm{PC}}=12.3 \mathrm{~Hz}, \mathrm{C}=\mathrm{O}\right), 172.27\left(\mathrm{~d},{ }^{3} J_{\mathrm{PC}}=16.5\right.$ $\mathrm{Hz}, \mathrm{C}=\mathrm{O}) .{ }^{31} \mathrm{P}$ NMR $\left(500.1 \mathrm{MHz}, \mathrm{CDCl}_{3}\right): \delta 23.48\left(\mathrm{Ph}_{3} \mathrm{P}^{+}-\mathrm{C}\right)$. 
Minor isomer (E) (3g). (38\%), ${ }^{1} \mathrm{H}$ NMR (500.1 MHz, $\left.\mathrm{CDCl}_{3}\right): \delta 1.05\left(3 \mathrm{H}, \mathrm{t},{ }^{3} J_{\mathrm{HH}}=7.0 \mathrm{~Hz}\right.$, $\left.\mathrm{CH}_{3}\right), 1.32\left(3 \mathrm{H}, \mathrm{t},{ }^{3} \mathrm{~J}_{\mathrm{HH}} 6.9 \mathrm{~Hz}, \mathrm{CH}_{3}\right), 3.71$ and $4.39\left(4 \mathrm{H}, 2 \mathrm{~m}, 2 \mathrm{OCH}_{2}\right), 4.69\left(1 \mathrm{H}, \mathrm{d},{ }^{3} J_{\mathrm{PH}}=19.4\right.$ $\mathrm{Hz}, \mathrm{P}=\mathrm{C}-\mathrm{CH}), 6.84(\mathrm{~s}, \mathrm{~N}=\mathrm{CH}), 6.74-8.67(24 \mathrm{H}, \mathrm{m}, 5 \mathrm{Ar}-\mathrm{H}) .{ }^{13} \mathrm{C} \mathrm{NMR}\left(500.1 \mathrm{MHz}, \mathrm{CDCl}_{3}\right): \delta$ 14.09 and $14.33\left(2 \mathrm{CH}_{3}\right), 42.06\left(\mathrm{~d},{ }^{1} J_{\mathrm{PC}}=133.6 \mathrm{~Hz}, \mathrm{P}=\mathrm{C}\right), 58.36$ and $61.60\left(2 \mathrm{OCH}_{2}\right), 65.90(\mathrm{~d}$, $\left.{ }^{2} J_{\mathrm{PC}}=15.9 \mathrm{~Hz}, \mathrm{P}=\mathrm{C}-\mathrm{CH}\right), 126.70\left(\mathrm{~d},{ }^{1} J_{\mathrm{PC}}=92.3 \mathrm{~Hz}, \mathrm{C}_{\mathrm{ipso}}\right), 128.59\left(\mathrm{~d},{ }^{3} J_{\mathrm{PC}}=12.3 \mathrm{~Hz}, \mathrm{C}_{\text {meta }}\right)$, $131.96\left(\mathrm{C}_{\text {para }}\right), 131.77\left(\mathrm{~d},{ }^{2} J_{\mathrm{PC}}=10.1 \mathrm{~Hz}, \mathrm{C}_{\text {ortho }}\right), 113.09,122.05,128.13,123.86,125.30,129.78$, 144.23, $145.98\left(8 \mathrm{C}_{\text {arom }}\right), 165.02(\mathrm{C}=\mathrm{N}), 169.57\left(\mathrm{~d},{ }^{2} J_{\mathrm{PC}}=16.7 \mathrm{~Hz}, \mathrm{C}=\mathrm{O}\right), 173.05\left(\mathrm{~d},{ }^{3} J_{\mathrm{PC}}=16.2\right.$ $\mathrm{Hz}, \mathrm{C}=\mathrm{O}) .{ }^{31} \mathrm{P}$ NMR $\left(202.4 \mathrm{MHz}, \mathrm{CDCl}_{3}\right): \delta 24.56\left(\mathrm{Ph}_{3} \mathrm{P}^{+}-\mathrm{C}\right)$.

Di-tert-butyl 2-(1-(4-nitrobenzylidene)-2-phenylhydrazine-1-yl)-3-(triphenylphosphoranylidene)butandioate (3h). Colorless crystals, yield $0.71 \mathrm{~g},(98 \%)$ mp 150-152 C, IR (KBr) $\left(v_{\max }, \mathrm{cm}^{-1}\right):(\mathrm{C}=\mathrm{O}) . \mathrm{MS}(\mathrm{m} / \mathrm{z}, \%): 729\left(\mathrm{M}^{+}, 1\right), 467$ (5), 411 (4), 310 (15), 277 (9), 262 (100), 241 (10), 183 (37), 108 (15), 92 (6), 77 (20). Anal. Calcd for $\mathrm{C}_{43} \mathrm{H}_{44} \mathrm{~N}_{3} \mathrm{O}_{6} \mathrm{P}$ (729.79): C, 70.77; H, 6.08; N, 5.76\% Found: C, 70.95; H, 6.11; N, 5.80\%

${ }^{1} \mathrm{H}$ NMR (500.1 MHz, $\left.\mathrm{CDCl}_{3}\right): \delta 0.87$ and $1.62\left(18 \mathrm{H}, 2 \mathrm{~s}, 2 \mathrm{CMe}_{3}\right), 4.47\left(1 \mathrm{H}, \mathrm{d},{ }^{3} J_{\mathrm{PH}}=18.3 \mathrm{~Hz}\right.$, $\mathrm{P}=\mathrm{C}-\mathrm{CH}), 6.84(\mathrm{~s}, \mathrm{~N}=\mathrm{CH}), 6.89-8.24(24 \mathrm{H}, \mathrm{m}, 5 \mathrm{Ar}-\mathrm{H}) .{ }^{13} \mathrm{C} \mathrm{NMR}\left(125.8 \mathrm{MHz}, \mathrm{CDCl}_{3}\right): \delta$ 27.98 and $28.25\left(2 \mathrm{CMe}_{3}\right), 39.64\left(\mathrm{~d},{ }^{1} J_{\mathrm{PC}}=125.2 \mathrm{~Hz}, \mathrm{P}=\mathrm{C}\right), 65.97\left(\mathrm{~d},{ }^{2} J_{\mathrm{PC}}=16.3 \mathrm{~Hz}, \mathrm{P}=\mathrm{C}-\mathrm{CH}\right)$, $126.98\left(\mathrm{~d},{ }^{1} \mathrm{~J}_{\mathrm{PC}} 91.6 \mathrm{~Hz}, \mathrm{C}_{\text {ipso }}\right), 128.37\left(\mathrm{~d},{ }^{3} \mathrm{~J}_{\mathrm{PC}} 12.1 \mathrm{~Hz}, \mathrm{C}_{\text {meta }}\right), 131.74\left(\mathrm{C}_{\mathrm{para}}\right), 133.65\left(\mathrm{~d},{ }^{2} J_{\mathrm{PC}}=\right.$ $\left.9.6 \mathrm{~Hz}, \mathrm{C}_{\text {ortho}}\right), 113.09,120.91,123.21,123.76,128.58,129.30,144.72,145.58\left(8 \mathrm{C}_{\text {arom }}\right), 164.44$ $(\mathrm{C}=\mathrm{N}), 167.64\left(\mathrm{~d},{ }^{2} J_{\mathrm{PC}}=12.0 \mathrm{~Hz}, \mathrm{C}=\mathrm{O}\right), 170.15\left(\mathrm{~d},{ }^{3} J_{\mathrm{PC}}=15.1 \mathrm{~Hz}, \mathrm{C}=\mathrm{O}\right) .{ }^{31} \mathrm{P}$ NMR $(202.4$ $\left.\mathrm{MHz}, \mathrm{CDCl}_{3}\right): \delta 23.09\left(\mathrm{Ph}_{3} \mathrm{P}^{+}-\mathrm{C}\right)$.

Diethyl 2-(1-(2-fluorobenzylidene)-2-phenylhydrazine-1-yl)-3-(triphenylphosphoranylidene)butandioate (3i). Colorless crystals, yield $0.63 \mathrm{~g}$, (98\%), mp 132-134 $\mathrm{C}$, IR (KBr) ( $v_{\max }$, $\left.\mathrm{cm}^{-1}\right): 1724,1646(\mathrm{C}=\mathrm{O}) . \mathrm{MS}(\mathrm{m} / \mathrm{z}, \%): 646\left(\mathrm{M}^{+}, 2\right), 433$ (3), 277 (65), 262 (100), 214 (57), 183 (42), 108 (16), 92 (19), 77 (23). Anal. Calcd for $\mathrm{C}_{39} \mathrm{H}_{36} \mathrm{FN}_{2} \mathrm{O}_{4} \mathrm{P}$ (646.68): C, 72.43; H, 5.61; N, 4.33\% Found: C, 72.42; H, 5.61; N, 4.28\%

Major isomer (Z) (3i). (68\%), ${ }^{1} \mathrm{H} \mathrm{NMR}\left(500.1 \mathrm{MHz}, \mathrm{CDCl}_{3}\right): \delta 0.39\left(3 \mathrm{H}, \mathrm{t},{ }^{3} \mathrm{~J}_{\mathrm{HH}}=7.0 \mathrm{~Hz}\right.$, $\left.\mathrm{CH}_{3}\right), 1.33\left(3 \mathrm{H}, \mathrm{t},{ }^{3} J_{\mathrm{HH}}=7.5 \mathrm{~Hz}, \mathrm{CH}_{3}\right), 3.53$ and $4.27\left(4 \mathrm{H}, 2 \mathrm{~m}, 2 \mathrm{OCH}_{2}\right), 4.63\left(1 \mathrm{H}, \mathrm{d},{ }^{3} J_{\mathrm{PH}}=\right.$ $17.1 \mathrm{~Hz}, \mathrm{P}=\mathrm{C}-\mathrm{CH}), 7.20(\mathrm{~s}, \mathrm{~N}=\mathrm{CH}), 6.25-7.96(24 \mathrm{H}, \mathrm{m}, 5 \mathrm{Ar}-\mathrm{H}) .{ }^{13} \mathrm{C} \mathrm{NMR}(125.8 \mathrm{MHz}$, $\left.\mathrm{CDCl}_{3}\right): \delta 14.10$ and $14.32\left(2 \mathrm{CH}_{3}\right), 40.17\left(\mathrm{~d},{ }^{1} J_{\mathrm{PC}}=124.6 \mathrm{~Hz}, \mathrm{P}=\mathrm{C}\right), 57.70$ and $61.38\left(2 \mathrm{OCH}_{2}\right)$, $64.08\left(\mathrm{~d},{ }^{2} J_{\mathrm{PC}}=15.2 \mathrm{~Hz}, \mathrm{P}=\mathrm{C}-\mathrm{CH}\right), 113.40\left(\mathrm{~d},{ }^{2} J_{\mathrm{FC}}=21.66 \mathrm{~Hz}, \mathrm{C}_{\text {ortho }}\right), 121.70\left(\mathrm{~d},{ }^{4} J_{\mathrm{FC}}=2.3 \mathrm{~Hz}\right.$, $\left.\mathrm{C}_{\mathrm{para}}\right), 126.39\left(\mathrm{~d},{ }^{1} J_{\mathrm{PC}}=91.7 \mathrm{~Hz}, \mathrm{C}_{\mathrm{ipso}}\right), 128.49\left(\mathrm{~d},{ }^{3} J_{\mathrm{PC}}=12.2 \mathrm{~Hz}, \mathrm{C}_{\text {meta }}\right), 129.48\left(\mathrm{~d},{ }^{3} J_{\mathrm{FC}}=8.4\right.$ $\left.\mathrm{Hz}, \mathrm{C}_{\text {meta }}\right), 129.97$ (d, $\left.{ }^{3} J_{\mathrm{FC}}=8.3 \mathrm{~Hz}, \mathrm{C}_{\text {meta }}\right), 131.77\left(\mathrm{C}_{\text {para }}\right), 133.57$ (d, $\left.{ }^{2} J_{\mathrm{PC}}=9.5 \mathrm{~Hz}, \mathrm{C}_{\text {ortho }}\right)$, 114.82, 120.14, 129.24, $145.08(4 \mathrm{C}, \mathrm{Ph}-\mathrm{N}), 163.04\left(\mathrm{~d},{ }^{1} J_{\mathrm{FC}}=243.8 \mathrm{~Hz}, \mathrm{C}_{\mathrm{ipso}}\right), 165.00(\mathrm{C}=\mathrm{N})$, $168.45\left(\mathrm{~d},{ }^{2} J_{\mathrm{PC}}=12.6 \mathrm{~Hz}, \mathrm{C}=\mathrm{O}\right), 172.56\left(\mathrm{~d},{ }^{3} J_{\mathrm{PC}}=15.9 \mathrm{~Hz}, \mathrm{C}=\mathrm{O}\right) .{ }^{31} \mathrm{P} \mathrm{NMR}(202.4 \mathrm{MHz}$, $\left.\mathrm{CDCl}_{3}\right): \delta 23.39\left(\mathrm{Ph}_{3} \mathrm{P}^{+}-\mathrm{C}\right)$.

Minor isomer (E) (3i). (32\%), ${ }^{1} \mathrm{H}$ NMR (500.1 MHz, $\left.\mathrm{CDCl}_{3}\right): \delta 1.09\left(3 \mathrm{H}, \mathrm{t},{ }^{3} J_{\mathrm{HH}}=7.0 \mathrm{~Hz}\right.$, $\left.\mathrm{CH}_{3}\right), 1.36\left(3 \mathrm{H}, \mathrm{t},{ }^{3} J_{\mathrm{HH}}=7.2 \mathrm{~Hz}, \mathrm{CH}_{3}\right), 3.81$ and $4.43\left(4 \mathrm{H}, 2 \mathrm{~m}, 2 \mathrm{OCH}_{2}\right), 4.65\left(1 \mathrm{H}, \mathrm{d},{ }^{3} J_{\mathrm{PH}}=\right.$ $19.4 \mathrm{~Hz}, \mathrm{P}=\mathrm{C}-\mathrm{CH}), 7.20(\mathrm{~s}, \mathrm{~N}=\mathrm{CH}), 6.25-7.96(24 \mathrm{H}, \mathrm{m}, 5 \mathrm{Ar}-\mathrm{H}) .{ }^{13} \mathrm{C} \mathrm{NMR}(500.1 \mathrm{MHz}$, $\left.\mathrm{CDCl}_{3}\right): \delta 13.98$ and $14.71\left(2 \mathrm{CH}_{3}\right), 42.26\left(\mathrm{~d},{ }^{1} J_{\mathrm{PC}}=133.7 \mathrm{~Hz}, \mathrm{P}=\mathrm{C}\right), 58.22$ and $60.90\left(2 \mathrm{OCH}_{2}\right)$, $66.45\left(\mathrm{~d},{ }^{2} J_{\mathrm{PC}}=17.9 \mathrm{~Hz}, \mathrm{P}=\mathrm{C}-\mathrm{CH}\right), 113.16\left(\mathrm{~d},{ }^{2} J_{\mathrm{FC}}=21.7 \mathrm{~Hz}, \mathrm{C}_{\text {ortho }}\right), 122.03\left(\mathrm{~d},{ }^{4} J_{\mathrm{FC}}=2.5 \mathrm{~Hz}\right.$, 
$\left.\mathrm{C}_{\mathrm{para}}\right), 126.59\left(\mathrm{~d},{ }^{1} J_{\mathrm{PC}}=91.7 \mathrm{~Hz}, \mathrm{C}_{\mathrm{ipso}}\right), 128.60\left(\mathrm{~d},{ }^{3} J_{\mathrm{PC}}=12.2 \mathrm{~Hz}, \mathrm{C}_{\mathrm{meta}}\right), 129.46\left(\mathrm{~d},{ }^{3} J_{\mathrm{FC}}=8.4\right.$ $\left.\mathrm{Hz}, \mathrm{C}_{\text {meta }}\right), 129.98\left(\mathrm{~d},{ }^{3} J_{\mathrm{FC}}=8.3 \mathrm{~Hz}, \mathrm{C}_{\text {meta }}\right), 131.93\left(\mathrm{C}_{\text {para }}\right), 133.72\left(\mathrm{~d},{ }^{2} J_{\mathrm{PC}}=9.7 \mathrm{~Hz}, \mathrm{C}_{\text {ortho }}\right)$, 115.11, 121.50, 129.79, $144.47(4 \mathrm{C}, \mathrm{Ph}-\mathrm{N}), 163.11\left(\mathrm{~d},{ }^{1} J_{\mathrm{FC}}=245.2 \mathrm{~Hz}, \mathrm{C}_{\mathrm{ipso}}\right), 165.00(\mathrm{C}=\mathrm{N})$, $169.61\left(\mathrm{~d},{ }^{2} J_{\mathrm{PC}}=17.6 \mathrm{~Hz}, \mathrm{C}=\mathrm{O}\right), 173.06\left(\mathrm{~d},{ }^{3} J_{\mathrm{PC}}=15.6 \mathrm{~Hz}, \mathrm{C}=\mathrm{O}\right) .{ }^{31} \mathrm{P} \mathrm{NMR}(202.4 \mathrm{MHz}$, $\left.\mathrm{CDCl}_{3}\right): \delta 24.29\left(\mathrm{Ph}_{3} \mathrm{P}^{+}-\mathrm{C}\right)$.

Dimethyl 2-(1-(2,4-dichlorobenzylidene)-2-phenylhydrazine-1-yl)-3-(triphenylphosphoranylidene)butandioate (3j). Colorless crystals, yield $0.65 \mathrm{~g},(98 \%), \mathrm{mp} 143-145^{\circ} \mathrm{C}, \mathrm{IR}(\mathrm{KBr})$ $\left(v_{\max }, \mathrm{cm}^{-1}\right): 1750,1648(\mathrm{C}=\mathrm{O}) . \mathrm{MS}(\mathrm{m} / \mathrm{z}, \%): 669\left(\mathrm{M}^{+}, 5\right), 405$ (8), 390 (17), 331 (30), 277 (100), 262 (87), 183 (87), 108 (29), 92 (23), 77 (76). Anal. Calcd for $\mathrm{C}_{37} \mathrm{H}_{31} \mathrm{ClN}_{2} \mathrm{O}_{4} \mathrm{P}$ (669.53): C, 66.37; H, 4.67; N, 4.18\% Found: C, 66.42; H, 4.76; N, 4.22\%

Major isomer (Z) (3j). (72\%), ${ }^{1} \mathrm{H}$ NMR (500.1 MHz, $\left.\mathrm{CDCl}_{3}\right): \delta 3.07$ and $3.88(6 \mathrm{H}, 2 \mathrm{~s}, 2$ $\left.\mathrm{OCH}_{3}\right), 4.62\left(1 \mathrm{H}, \mathrm{d},{ }^{3} J_{\mathrm{PH}}=16.5 \mathrm{~Hz}, \mathrm{P}=\mathrm{C}-\mathrm{CH}\right), 6.93(\mathrm{~s}, \mathrm{~N}=\mathrm{CH}), 6.93-8.00(23 \mathrm{H}, \mathrm{m}, 5 \mathrm{Ar}-\mathrm{H})$. ${ }^{13} \mathrm{C} \mathrm{NMR}\left(125.8 \mathrm{MHz}, \mathrm{CDCl}_{3}\right): \delta 40.27\left(\mathrm{~d},{ }^{1} J_{\mathrm{PC}}=125.0 \mathrm{~Hz}, \mathrm{P}=\mathrm{C}\right), 49.20$ and $52.78\left(2 \mathrm{OCH}_{3}\right)$, $63.81\left(\mathrm{~d},{ }^{2} J_{\mathrm{PC}}=15.0 \mathrm{~Hz}, \mathrm{P}=\mathrm{C}-\mathrm{CH}\right), 126.13\left(\mathrm{~d},{ }^{1} J_{\mathrm{PC}}=91.7 \mathrm{~Hz}, \mathrm{C}_{\mathrm{ipso}}\right), 128.67\left(\mathrm{~d},{ }^{3} J_{\mathrm{PC}}=12.3 \mathrm{~Hz}\right.$, $\left.\mathrm{C}_{\text {meta }}\right), 131.97\left(\mathrm{C}_{\text {para }}\right), 133.45\left(\mathrm{~d},{ }^{2} J_{\mathrm{PC}}=9.7 \mathrm{~Hz}, \mathrm{C}_{\text {ortho }}\right), 122.95,123.45,126.84,128.17,128.59$, 129.15, 133.23, 144.82, (8 C arom), $165.83(\mathrm{C}=\mathrm{N}), 168.92\left(\mathrm{~d},{ }^{2} J_{\mathrm{PC}}=12.6 \mathrm{~Hz}, \mathrm{C}=\mathrm{O}\right), 172.48(\mathrm{~d}$, $\left.{ }^{3} J_{\mathrm{PC}}=16.0 \mathrm{~Hz}, \mathrm{C}=\mathrm{O}\right) .{ }^{31} \mathrm{P}$ NMR $\left(202.4 \mathrm{MHz}, \mathrm{CDCl}_{3}\right): \delta 23.51\left(\mathrm{Ph}_{3} \mathrm{P}^{+}-\mathrm{C}\right)$.

Minor isomer (E) (3j). (28\%), ${ }^{1} \mathrm{H}$ NMR (500.1 MHz, $\left.\mathrm{CDCl}_{3}\right): \delta 3.42$ and $3.80(6 \mathrm{H}, 2 \mathrm{~s}, 2$ $\left.\mathrm{OCH}_{3}\right), 4.68\left(1 \mathrm{H}, \mathrm{d},{ }^{3} \mathrm{~J}_{\mathrm{PH}}=17.4 \mathrm{~Hz}, \mathrm{P}=\mathrm{C}-\mathrm{CH}\right), 6.93(\mathrm{~s}, \mathrm{~N}=\mathrm{CH}), 6.93-8.00(23 \mathrm{H}, \mathrm{m}, 5 \mathrm{Ar}-\mathrm{H})$. ${ }^{13} \mathrm{C} \mathrm{NMR}\left(125.8 \mathrm{MHz}, \mathrm{CDCl}_{3}\right): \delta 42.06\left(\mathrm{~d},{ }^{1} J_{\mathrm{PC}}=133.7 \mathrm{~Hz}, \mathrm{P}=\mathrm{C}\right), 50.06$ and $52.32\left(2 \mathrm{OCH}_{3}\right)$, $66.35\left(\mathrm{~d},{ }^{2} J_{\mathrm{PC}}=14.8 \mathrm{~Hz}, \mathrm{P}=\mathrm{C}-\mathrm{CH}\right), 126.00\left(\mathrm{~d},{ }^{1} J_{\mathrm{PC}}=92.0 \mathrm{~Hz}, \mathrm{C}_{\mathrm{ipso}}\right), 128.83\left(\mathrm{~d},{ }^{3} J_{\mathrm{PC}}=11.6 \mathrm{~Hz}\right.$, $\left.\mathrm{C}_{\text {meta }}\right), 132.05\left(\mathrm{C}_{\text {para }}\right), 133.71\left(\mathrm{~d},{ }^{2} J_{\mathrm{PC}}=9.8 \mathrm{~Hz}, \mathrm{C}_{\text {ortho }}\right), 112.76,123.53,126.40,128.17,128.33$, 128.43, 132.12, 143.23, (8 $\left.\mathrm{C}_{\text {arom }}\right), 165.83(\mathrm{C}=\mathrm{N}), 169.80\left(\mathrm{~d},{ }^{2} J_{\mathrm{PC}}=17.8 \mathrm{~Hz}, \mathrm{C}=\mathrm{O}\right), 173.21(\mathrm{~d}$, $\left.{ }^{3} J_{\mathrm{PC}}=14.9 \mathrm{~Hz}, \mathrm{C}=\mathrm{O}\right) .{ }^{31} \mathrm{P} \mathrm{NMR}\left(202.4 \mathrm{MHz}, \mathrm{CDCl}_{3}\right): \delta 23.91\left(\mathrm{Ph}_{3} \mathrm{P}^{+}-\mathrm{C}\right)$.

Diethyl 2-(1-(4-fluorobenzylidene)-2-phenylhydrazine-1-yl)-3-(triphenylphosphor anylidene)butandioate (3k). Colorless crystals, yield $0.63 \mathrm{~g},(98 \%), \mathrm{mp} 136-138 \mathrm{C}, \mathrm{IR}(\mathrm{KBr})$ $\left(v_{\max }, \mathrm{cm}^{-1}\right): 1729,1646(\mathrm{C}=\mathrm{O}) . \mathrm{MS}(\mathrm{m} / \mathrm{z}, \%): 646\left(\mathrm{M}^{+}, 8\right), 433(5), 277(100), 262$ (52), 214 (59), 183 (55), 108 (19), 92 (26), 77 (69). Anal. Calcd for $\mathrm{C}_{39} \mathrm{H}_{36} \mathrm{CFN}_{2} \mathrm{O}_{4} \mathrm{P}$ (646.68): C, 72.43; H, $5.61 ; \mathrm{N}, 4.33 \%$ Found: C, 72.50; H, 5.60; N, 4.27\%

Major isomer (Z) (3k). (70\%), ${ }^{1} \mathrm{H} \mathrm{NMR}\left(500.1 \mathrm{MHz}, \mathrm{CDCl}_{3}\right): \delta 0.39\left(3 \mathrm{H}, \mathrm{t},{ }^{3} J_{\mathrm{HH}}=7.0 \mathrm{~Hz}\right.$, $\left.\mathrm{CH}_{3}\right), 1.30\left(3 \mathrm{H}, \mathrm{t},{ }^{3} J_{\mathrm{HH}}=7.5 \mathrm{~Hz}, \mathrm{CH}_{3}\right), 3.52$ and $3.78\left(4 \mathrm{H}, 2 \mathrm{~m}, 2 \mathrm{OCH}_{2}\right), 4.63\left(1 \mathrm{H}, \mathrm{d},{ }^{3} J_{\mathrm{PH}}=\right.$ $17.0 \mathrm{~Hz}, \mathrm{P}=\mathrm{C}-\mathrm{CH}), 7.78(\mathrm{~s}, \mathrm{~N}=\mathrm{CH}), 6.24-7.72(24 \mathrm{H}, \mathrm{m}, 5 \mathrm{Ar}-\mathrm{H}) .{ }^{13} \mathrm{C} \mathrm{NMR}(125.8 \mathrm{MHz}$, $\left.\mathrm{CDCl}_{3}\right): \delta 14.10$ and $14.32\left(2 \mathrm{CH}_{3}\right), 40.20\left(\mathrm{~d},{ }^{1} J_{\mathrm{PC}}=124.4 \mathrm{~Hz}, \mathrm{P}=\mathrm{C}\right), 57.68$ and $61.36\left(2 \mathrm{OCH}_{2}\right)$, $63.79\left(\mathrm{~d},{ }^{2} \mathrm{~J}_{\mathrm{PC}} 14.3 \mathrm{~Hz}, \mathrm{P}=\mathrm{C}-\mathrm{CH}\right), 115.05\left(\mathrm{~d},{ }^{2} \mathrm{~J}_{\mathrm{FC}} 21.8 \mathrm{~Hz}, \mathrm{C}_{\text {ortho }}\right), 121.79\left(\mathrm{~d},{ }^{4} \mathrm{~J}_{\mathrm{FC}}=2.0 \mathrm{~Hz}, \mathrm{C}_{\text {para }}\right)$, $126.39\left(\mathrm{~d},{ }^{1} J_{\mathrm{PC}}=91.7 \mathrm{~Hz}, \mathrm{C}_{\mathrm{ipso}}\right), 128.49\left(\mathrm{~d},{ }^{3} J_{\mathrm{PC}}=12.2 \mathrm{~Hz}, \mathrm{C}_{\text {meta }}\right), 129.23\left(\mathrm{~d},{ }^{3} J_{\mathrm{FC}}=8.1 \mathrm{~Hz}\right.$, $\left.\mathrm{C}_{\text {meta }}\right), 131.77\left(\mathrm{C}_{\text {para }}\right), 133.57\left(\mathrm{~d},{ }^{2} J_{\mathrm{PC}}=9.5 \mathrm{~Hz}, \mathrm{C}_{\text {ipso }}\right), 115.73,119.93,128.17,145.43(4 \mathrm{C}, \mathrm{Ph}-$ $\mathrm{N}), 162.76\left(\mathrm{~d},{ }^{1} J_{\mathrm{FC}}=248.2 \mathrm{~Hz}, \mathrm{C}_{\mathrm{ipso}}\right), 165.01(\mathrm{C}=\mathrm{N}), 168.47\left(\mathrm{~d},{ }^{2} J_{\mathrm{PC}}=12.5 \mathrm{~Hz}, \mathrm{C}=\mathrm{O}\right), 172.73(\mathrm{~d}$, $\left.{ }^{3} J_{\mathrm{PC}}=16.0 \mathrm{~Hz}, \mathrm{C}=\mathrm{O}\right) .{ }^{31} \mathrm{P} \mathrm{NMR}\left(202.4 \mathrm{MHz} ; \mathrm{CDCl}_{3}\right): \delta 23.38\left(\mathrm{Ph}_{3} \mathrm{P}^{+}-\mathrm{C}\right)$.

Minor isomer (E) (3k). (30\%), ${ }^{1} \mathrm{H}$ NMR $\left(500.1 \mathrm{MHz}, \mathrm{CDCl}_{3}\right): \delta 1.11\left(3 \mathrm{H}, \mathrm{t},{ }^{3} J_{\mathrm{HH}}=6.9 \mathrm{~Hz}\right.$, $\left.\mathrm{CH}_{3}\right), 1.35\left(3 \mathrm{H}, \mathrm{t},{ }^{3} J_{\mathrm{HH}}=7.2 \mathrm{~Hz}, \mathrm{CH}_{3}\right), 4.27$ and $4.40\left(4 \mathrm{H}, 2 \mathrm{~m}, 2 \mathrm{OCH}_{2}\right), 4.64\left(1 \mathrm{H}, \mathrm{d},{ }^{3} J_{\mathrm{PH}}=\right.$ 
$19.5 \mathrm{~Hz}, \mathrm{P}=\mathrm{C}-\mathrm{CH}), 7.78(\mathrm{~s}, \mathrm{~N}=\mathrm{CH}), 6.24-7.72(24 \mathrm{H}, \mathrm{m}, 5 \mathrm{Ar}-\mathrm{H}) .{ }^{13} \mathrm{C} \mathrm{NMR}(125.8 \mathrm{MHz}$, $\left.\mathrm{CDCl}_{3}\right): \delta 13.99$ and $14.75\left(2 \mathrm{CH}_{3}\right), 41.80\left(\mathrm{~d},{ }^{1} \mathrm{~J}_{\mathrm{PC}}=133.6 \mathrm{~Hz}, \mathrm{P}=\mathrm{C}\right), 58.20$ and $60.86\left(2 \mathrm{OCH}_{2}\right)$, $65.90\left(\mathrm{~d},{ }^{2} J_{\mathrm{PC}}=16.1 \mathrm{~Hz}, \mathrm{P}=\mathrm{C}-\mathrm{CH}\right), 114.95\left(\mathrm{~d},{ }^{2} J_{\mathrm{FC}}=21.58 \mathrm{~Hz}, \mathrm{C}_{\mathrm{ortho}}\right), 122.28\left(\mathrm{~d},{ }^{4} J_{\mathrm{FC}}=2.0 \mathrm{~Hz}\right.$, $\left.\mathrm{C}_{\mathrm{para}}\right), 127.41\left(\mathrm{~d},{ }^{1} J_{\mathrm{PC}}=94.0 \mathrm{~Hz}, \mathrm{C}_{\mathrm{ipso}}\right), 128.56\left(\mathrm{~d},{ }^{3} J_{\mathrm{PC}}=12.0 \mathrm{~Hz}, \mathrm{C}_{\text {meta }}\right), 129.79\left(\mathrm{~d},{ }^{3} J_{\mathrm{FC}}=8.1\right.$ $\left.\mathrm{Hz}, \mathrm{C}_{\text {meta }}\right), 131.93$ ( $\left.\mathrm{C}_{\text {para }}\right), 133.72\left(\mathrm{~d},{ }^{2} J_{\mathrm{PC}}=12.6 \mathrm{~Hz}, \mathrm{C}_{\text {ortho }}\right), 115.44,122.61,128.07,144.71$ (4C, $\mathrm{Ph}-\mathrm{N}), 161.93\left(\mathrm{~d},{ }^{1} J_{\mathrm{FC}}=245.7 \mathrm{~Hz}, \mathrm{C}_{\mathrm{ipso}}\right), 165.01(\mathrm{C}=\mathrm{N}), 169.62\left(\mathrm{~d},{ }^{2} J_{\mathrm{PC}}=17.5 \mathrm{~Hz}, \mathrm{C}=\mathrm{O}\right)$, $173.11\left(\mathrm{~d},{ }^{3} \mathrm{~J}_{\mathrm{PC}}=17.0 \mathrm{~Hz}, \mathrm{C}=\mathrm{O}\right) .{ }^{31} \mathrm{P}$ NMR $\left(202.4 \mathrm{MHz} ; \mathrm{CDCl}_{3}\right): \delta 24.18\left(\mathrm{Ph}_{3} \mathrm{P}^{+}-\mathrm{C}\right)$.

\section{Acknowledgements}

We gratefully acknowledge financial support from the Research Council of the University of Sistan \& Baluchestan.

\section{References}

1. Corbridge, D. E. C. Phosphorus: An Outline of The Chemistry, Biochemistry and Uses, Elsevier: Amsterdam, $5^{\text {th }}$ Edn, 1995.

2. Murphy, P. J. Organophosphorus Reagents, Oxford University Press: Oxford, $1^{\text {st }}$ Ed, 2004.

3. Engel, R.; Cohen, J. I. Synthesis of Carbon-Phosphorus Bonds, CRC Press: Boca Raton, FL, $2^{\text {nd }} \mathrm{Ed}, 2004$.

4. Johnson, A. W. Ylide Chemistry, Academic Press: London, 1966.

5. Wittig, G. Science 1980, 210, 500.

6. Kolodiazhnyi, O. I. Phosphorus Ylides: Chemistry and Application in Organic Synthesis, Wiley-VCH: Weinheim, 1999.

7. Cadogan, J. I. G. Organophosphorus Reagents in Organic Synthesis, Academic Press: New York, 1979.

8. Hudson, H. R. Nucleophilic Reactions of Phosphines, ch. 11 in The Chemistry of Organophosphorus Compounds, Primary, Secondary, and Tertiary Phosphates and Heterocyclic Organophosphorus III Compounds, Hartely, F. R., Ed., Wiley: New York, 1990.

9. Maryanoff, B. E.; Rietz, A. B. Chem. Rev. 1989, 89, 863.

10. Nicolaou, K. C.; Harter, M. W.; Gunzner, J. L.; Nadin, A. Liebigs Ann. 1997, 7,1283.

11. Shen, Y. Acc. Chem. Res. 1998, 31, 584.

12. Quin, L. D. A Guide to Organophosphorus Chemistry, Wiley-Interscience: New York, 2000.

13. Yavari, I.; Esmaili, A. A. J. Chem. Res. 1998, 714.

14. Maghsoodlou, M. T.; Hazeri, N.; Habibi Khorassani, S. M.; Nassiri, M.; Marandi, G.; Afshari, G.; Niroumand, U. J. Sulfur. Chem. 2005, 26, 261.

15. Islami, M. R.; Mollazehi, F.; Badiei, A.; Sheibani, H. Arkivoc 2005, xv, 25. 
16. Maghsoodlou, M. T.; Hazeri, N.; Habibi Khorassani, S. M.; Afshari, G.; Nassiri, M. J. Chem. Res. (S). 2005, 727.

17. Yavari, I.; Islami, M. R. J. Chem. Res. 1998, 166.

18. Yavari, I.; Esmaili, A.; Asghari, S.; Bijanzadeh, H. R. J. Chem. Res. 1999, 368.

19. Hazeri, N.; Habibi Khorassani, S. M.; Maghsoodlou, M. T.; Marandi, G.; Nassiri, M.; Gulam-Shahzadeh, A. J. Chem. Res. (S). 2006, 215.

20. Maghsoodlou, M. T.; Saghatforoush, L.; Aminkhani, A.; Marandi, G.; Kabiri, R. J. Sulfur. Chem. 2006, 27, 583.

21. Maghsoodlou, M. T.; Hazeri, N.; Habibi Khorassani, S. M.; Kakaei, R.; Nassiri, M.; Marandi, G.; Moeeni, Z.; Niromand, U.; Eskandari-Torbaghan, Z. Phosphorus, Sulfur, and Silicon. 2006, 181, 865.

22. Kalantari, M.; Islami, M. R.; Hassani, Z.; Saidi, K. Arkivoc 2006, x, 55.

23. Hassani, Z.; Islami, M. R.; Sheibani, H.; Kalantari, M.; Saidi, K. Arkivoc 2006, i, 89.

24. Maghsoodlou, M. T.; Hazeri, N.; Habibi Khorassani, S. M.; Saghatforoush, L.; Rofouei, M. K.; Rezaie, M. Arkivoc 2006, xiii, 117.

25. Maghsoodlou, M. T.; Habibi Khorassani, S. M.; Hazeri, N.; Nassiri, M. Phosphorus, Sulfur, and Silicon. 2006, 181, 1363.

26. Hazeri, N.; Maghsoodlou, M. T.; Habibi Khorassani, S. M.; Nassiri, M.; Afarini, Z. J. Chem. Res. 2008, 97.

27. Maghsoodlou, M. T.; Habibi Khorassani, S. M.; Nassiri, M.; Adhamdoust, S. R.; Salehzadeh, J. J. Chem. Res. 2008, 79.

28. Esmaili, A. A.; Ghereghloo, M.; Islami, M. R.; Bijanzadeh, H. R. Tetrahedron 2003, 59, 4785.

29. Bestmann, H. J.; Joachim, G.; Lengyel, T.; Oth, J. F.; Merenyi, R.; Weitkamp, H. Tetrahedron Lett. 1966, 3355.

30. Islami, M. R.; Yavari, I.; Tikdari, A. M.; Ebrahimi, L.; Razee, S.; Bijanzadeh, H. R. Russ. Chem. Bull. 2002, 51, 2244.

31. Maghsoodlou, M. T.; Shaterian, H. R.; Marandi, G.; Shahraki Poor, F.; Salehzadeh, J. Arkivoc 2008, xiii, 218.

32. Furniss, B. S.; Hannaford, A. J.; Smith, P. W. G.; Tatchell, A. R. In: Vogel's, Text Book of Practical Organic Chemistry, $5^{\text {th }}$ (ed) Wiley: New York, 1989, p. 478. 